\title{
EduBridge Social Bridging Social Networks and Learning Management Systems
}

\author{
Luciana Oliveira ${ }^{1}$ and Álvaro Figueira ${ }^{2}$ \\ ${ }^{1}$ CICE/ISCAP \& INESC TEC, Polythecnic of Porto, Rua Jaime Lopes Amorim, Porto, Portugal \\ ${ }^{2}$ CRACS/INESC TEC, University of Porto, Porto, Portugal
}

Keywords: Social Student Relationship Management, Educational Communication, Learning Management System, Facebook Groups, Moodle.

\begin{abstract}
The exponential growth of social media usage and the integration of digital natives in Higher Education Institutions (HEI) have been posing new challenges to both traditional and technology-mediated learning environments. Nowadays social media plays an important, if not central, role in society, for professional and personal purposes. However, it's important to highlight that in the mind of a digital native, social media is not just a tool, it is a place that is as real and as natural as any real-life world place where formal/informal social interactions happen. Still, formal higher education contexts are still mostly imprisoned in locked up institutional Learning Management Systems (LMS), while a new world of social connections grows and develops itself outside schools. One of the main reasons we believe to be persisting in the origin of the matter is the absence of a suitable management, monitoring and analysis tools to legitimize and to efficiently manage the relationship with students in social networks. In this paper we discuss the growing relevance of the "Social Student Relationship Management" concept and introduce the EduBridge Social system, which aims at connecting the most commonly used LMS, Moodle, and the most popular social network, Facebook.
\end{abstract}

\section{$1-$ INTRODUCTION}

The tendency to value and strategically frame the development of social interactions transcends the transactional and organizational needs and environment or organizations at large, and of Higher Education institutions in particular. It is a clear reflexion on how communication has been evolving since the growth and intensification of the use of social media platforms, that impacts on how society at large relates to organizations and, on a micro level, on how individuals relate to each other. It reveals a strengthening of the relationships between publics and organizations and also the intensification of twoway communication streams. In fact, social media has been redefining how we relate to each other as humans and how we as humans relate to the organizations that serve us (Reuben, 2008). It has been imposing a two-way dialog that brings people together to discover and share information, share ideas and build communities of people that share the same interests.

The concept of Social Student Relationship Management (Social SRM) consists of a particularization of the Social CRM concept, applied to the relationship between educational providers (such as schools, universities and training centres) and students supported by social media platforms.

Social SRM aims at amplifying the formal and informal bonds between students and schools, expanding their interactions into social conversations, and strengthening educational ties through the development of collaborative conversations that provide mutually beneficial value and that, ultimately, allows for the growth of social and educational communities. It places organizations, teachers and staff in student's digital natural environment, engaging them in personalized dialogs that reinforce students' role as empowered participatory individuals in organizational strategic development and also in the management of their learning opportunities.

The educational dimension of Social SRM comprises the set of relationship and interactions between teachers and students, in social media, in the domain of teaching-learning communication, in the classroom administration and in the students' social integration. As a subsystem of internal organizational 
communication it brings organizational culture into the relationship and builds upon it, since "students' experiences depend on their social class backgrounds, the responses of school staff to their behaviour within schools, and the actions of students and staff that create school cultures" (Parsons, 2014).

We believe that the quality of the communication in a learning system significantly impacts on its efficiency and that the state-of-the-art information technologies, mainly social media, offers unlimited resources for the communication's streamlining and diversification of formal and informal learning (Dorin, 2013).

Social media has been boosting educators to drive formal learning contexts into social networks aiming at the development of learning communities that allow for collaborative exploration and reflection of ideas, in a cooperative and supportive atmosphere (Palloff and Pratt, 2007). Besides offering participants the potential to benefit from a ready source of peer support it also provides the necessary emotional support (Bruckman, 2006) to allow for communities' social inclusion.

In the next section of this paper we present the background of the Social SRM concept and the educational domains of its application, since our main focus resides on the communicative / dialogical nature of the interactions happening inside the learning communities.

In section 3 a case study focused on the educational use of Facebook groups is presented, consolidating the main dimensions of the Social SRM concept, where the need for a management / interaction system is raised. Needs addressed in this paper are essentially concerned with systems' integration and social media information retrieval. This is our key priority for the first stage of development of the system presented in section 4.

In section 4 we introduce and present the EduBridge Social system which aims to bridge educational environments with social networks. We begin by describing the system's architecture, how it integrates with Moodle and Facebook, and how authentication is managed. In section 5 we detail the main aspects of the system's interface and the system's relevance / contribution in terms of potential to improve the management of the educational environment for teachers. Finally, in the last section we present our conclusions and future work.

\section{BACKGROUNG ON SOCIAL SRM}

The concept of Social SRM builds upon previous research on the use of Facebook groups in education, as reported by Oliveira (2015). In order to fully clarify it, it is essential to present its three main components.

\subsection{Teaching-learning Communication}

In this domain communication serves as a vehicle for reaching educational goals. It encompasses formal educational communication, which is sustained by a curriculum and pedagogic model with clear indications of contents, methods, requirements and assessment, and the informal educational communication, which is not institutionalized, methodical, structured, intentional and sustained by a previous definition of pedagogic goals. Informal educational communication is more prone to social media environments, thus it's important to understand that the absence of a curriculum and pedagogic model doesn't annul its educational potential. The spontaneous, simple conversation and group discussion that characterizes informal communication may produce educational effects, when contributing to behavioural changes in individuals.

\subsection{Classroom Administration}

This domain is in direct dependency of the organizational administrative communication that regulates learning tasks, schedules and procedures. Social media has proven to be an excellent tool for classroom administration, in terms of processes clarification and celerity, student responsiveness and time economy. This is heavily sustained by the amount of time and attention students dedicate to social media.

\subsection{Student Social Integration}

This dimension is aimed at fostering the formation of the sense of belonging and ultimately the development of a learning community. It consists of the most pure form of social interaction, relying heavily on the establishment of meaningful conversations that allow for the development of social and emotional bonds. From the teachers' point of view, and as a community builder/manager, it requires an engaging, motivational, cooperative and 
personal/informal communication approach that is favoured in social media environments.

Educational Social SRM comprises a set of communication domains that aim to foster student integration and avoid disengagement or dropout. It contributes to setting a welcoming school climate prone to success and completion, which has been defined as "...the quality and character of school life experiences and reflects norms, goals, values interpersonal relationships, teaching, learning and leadership practices, and organizational structures" (Bryant, 2013). For instance, in a blended learning environment, social media provides two-way exchanges between the classroom and the virtual interaction environment in the relationship building process - both environments being interchangeably reinforced.

\section{FACEBOOK GROUPS IN EDUCATION}

In this section we present evidence on previous research (Oliveira 2015) conducted on the use of Facebook groups, as an extension of the classroom teacher-student relationship, into a more dynamic and informal environment.

The aim of this case study was to discover, investigate and assess the three components of the educational dimension of Social SRM mentioned in the previous section. Research also allowed to obtain insights on the benefits, challenges and tech needs for a broader implementation of this methodology.

The study was carried out during two semesters, in three different subjects from the first, second and third year from two different courses. The courses were held in traditional classroom, with Moodle support. A Facebook group was created for each class and the students were invited to join. Though participation was not mandatory, all of the students having a Facebook profile joined the groups, except for 3 students who didn't own a Facebook profile and didn't wish to own one. Among the three classes there were 99 students (grouped in 52, 34 and 13 participants) and one teacher interacting in three separate Facebook groups. Facebook groups were introduced to students as a complimentary support platform in the first class of the semester and not as a replacement for the institutional LMS, as Wang proposes (Wang et al., 2012).

We must stress that the focus resided on the communicative / dialogical nature of the interactions happening inside the learning communities, mainly because there was no particular objective assigned to participants when these communities were formed. Students were invited to join a "support community" where anything could happen, in order to assure that dialogue and relationships were spontaneous and selfmotivated, as already defended by some authors (Deng \& Tavares, 2013). Students were encouraged to bring any subject they found relevant to the group.

All the groups' interactions were retrieved from Facebook, using the available API. In this process, the following fields were collected on each groups feed: post id, post author, post message, post type and corresponding \#comments and \#likes. The posts were classified in 5 main subject categories, which were created to fit the natural and dynamic discussions that emerged in the groups: (1) course administration, (2) posts related to course contents / curriculum, (3) course unrelated personal interests / projects, (4) posts related to academic training and personal development and (5) social messages. Additionally, in order to better understand these results and to dig into personal perspectives, students were surveyed.

Concerning the domain of educational communication and its specificities in the social media environment, in the case study, the three Social SRM dimensions were not equitably detected: messages related to teaching-learning and course administration rounded up to $40 \%$ each and messages aimed at the student social integration rounded only up to $20 \%$. Still, it was possible to identify several positive impacts from the implementation of the above mentioned methodology, which were consolidated with survey responses.

When asked if they would like to connect and communicate with other teachers on Facebook, 89\% of the students responded affirmatively. When asked why, these mention mainly: the celerity in responses/clarification of doubts, the increased interaction with the teacher, the improvement of the communication with the teacher, the easiness to keep up and the increased visibility/sense of presence of course activities, discussions and administration, the enlarged team spirit and the fact that teachers post relevant content in course's scientific domain.

Students were also questioned about their perspectives on the social relationship they maintain with their teachers, in order to provide a fuller picture on the importance of the social dimension of this relationship. $94 \%$ of the students believes that a good social relationship between teacher and students is fundamental for academic success and that teachers do not invest enough on the formation of these relationships (74\%). 93\% of the students would like 
other teachers to use Facebook for educational and social purposes, increasing teacher-student interactions.

When asked about the Facebook group usefulness, effectiveness, communication and relationship potential, students reported that it contributes to a better working relationship with peers $(96 \%)$, it increases the socialization potential among classmates $(92 \%)$, it provides a better social and professional relationship with the teacher $(96 \%)$, it increase their motivation to learn (84\%) and that they feel more actively participating and engaged in course activities $(90 \%)$, that their peers value their contributions in the Facebook group (89\%) and that, in the Facebook group, the class works as cooperative team $(91 \%)$. Engagement in Facebook has also been shown to be closely related to an increase in college students' social capital, especially for those with low self-esteem and life satisfaction (Deng \& Tavares, 2013).

On the teacher perspective, the extension of classroom communication into social media can provide real benefits, but it can also pose some challenges. Using Facebook groups to answer students' questions and doubts can be real timeeffective, since clarification becomes widely available for everyone. When it comes to requiring student's attention or immediate actions, social media offers excellent celerity as students respond almost immediately. Facebook groups also allowed shy/introverted students to become more actively engaged in discussions and, though it may be possible to discuss details associated to teacher-student privacy, it can be quite useful to understand the student general state of mind, general interests and social background context, which becomes accessible.

On the other hand, the teacher may face some challenges during the extension of classroom communication into social media, such as: compromising their privacy on the network, students expecting quicker responses and student active engagement may require a lot of moderation time. In these environments it is convenient to lower the level of relationship formality, which may conflict with a very formal classroom teacher attitude and/or intrapersonal communication style.

Cultural issues must also be raised. There is a profound heritage of formality and vertical relationships in HEI's cultural environment which may pose additional challenges to the proposed methodology. In order to address these challenges further research should focus on determining how informal should teacher-student interactions be on social media, providing clear guidelines resulting from the convergence of mutual expectations.

The conducted study also revealed that, in order to efficiently manage and assess social students' relationships, teachers need suitable monitoring and analysis tools/applications.

In our perspective, and based on students' and teachers' perceptions on the use of social networks as complimentary educational systems, the teacher is the agent facing the bigger challenges. In fact, students naturally and easily accommodate several dimensions of their lives in social networks (personal, professional, social, educational), and are very open to changes. However, on top of the previously mentioned challenges that teachers may face, there is also the need to institutionally and educationally legitimize the use of social networks, since some teachers refuse to adopt concurrent non-institutional systems, such as the school's LMS. This lays on top of deep cultural issues and on the organization's proneness to adopt/adjust to new technologies.

Given this scenery, we believe to be of the utmost importance to bridge between the well-established LMS and the also well-established social networks (ex. Facebook), taking advantage of pre-acquired habits, knowledge and sense of control, which is currently offered to teachers. We believe that providing the current LMS with insights and features that allow teachers to manage and perceive the dynamics of students' interactions on social media, to manage them and to make educational sense of them, may, in fact, facilitate and potentiate the inclusion of social networks on the best service of education.

At this stage, and in this paper, our main focus is to provide teachers with resources to validate the use of social media in their educational settings. Though research emerges at a quick pace, teachers' technology adoption pace and the process of integrating a wider variety of technologies in their pedagogical framework is considerably slower. In fact, despite the advances in research and the proven evidence of the benefits of the educational use of social media in education, it doesn't necessarily mean that there is a corresponding widespread adoption of it. Therefore, we believe that, at a first instance, it is necessary to gather teachers' consensus by fomenting a shift in practices (for instance, through teacher training) and secondly by providing a technological solution that assures the maintenance of the validity, security and institutional recognition of the required shift in pedagogical practices. Research outputs from the work of Oliveira (2015) were already converted into several teacher dissemination, training and demonstration initiatives, thus its outputs are being actively offered to educational professionals and 
actually being incorporated in their practices. In order to consolidate this process, an urgent technological development is crucial in order to facilitate the necessary shift, and this is why, for now, teachers are at the centre or our main concerns, since they are the main catalyst agents.

On the following section, we present the architecture of the EduBridge system, built fulfil the above mentioned needs.

\section{SYSTEM'S ARCHITECTURE}

Our proposed system bridges one learning management system with a social network. Our approach was to focus on the popular of these two systems. According to its popularity and maturity, we naturally picked Moodle and Facebook.

The proposed system is based on a set of Moodle blocks that share a communications engine and a database stored locally. The system's architecture is based on the model - view - control pattern, implemented through five modules. In this case, the 'view' is produced by each module. However, the 'control' is hierarchically spread through the top level-module down to the leaves modules. This organization is depicted in Figure 1. As a top layer we have the communications module (googleoauther 2) which establishes a connection between Moodle and Facebook through the Facebook's authentication mechanism, using the user's credentials. Then, the control is passed to the module fbgroups to retrieve the groups owned by the user. This module is also responsible for creating lists of users belonging to the groups and to set some counters for usage statistics. The module fbcomments retrieves all the posts and comments that were posted in timeline of the selected group. Finally, the modules fbstats and fbtotalstats present the local statistics, and global statistics, about the selected group or all the considered groups, respectively.

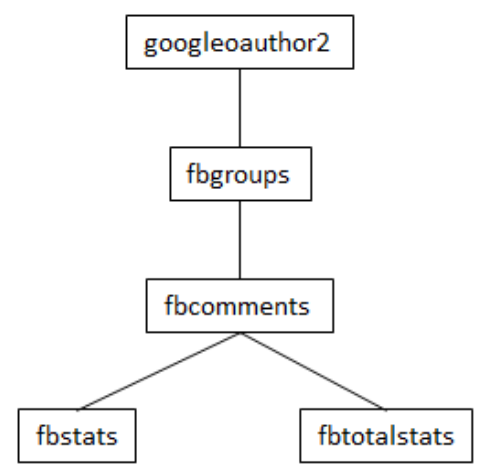

Figure 1: The system's 5 modules.
These modules were implemented as simple Moodle blocks. The reasons for this decision mainly rely on:

- It's easier to maintain modular code;

- Moodle blocks have enough capabilities to include code which is centrally administrated, and that can share a common access to specific tables in the database;

- The blocks positioning system is quite customizable in versions after Moodle 2.8 making it simpler to be adapted to the visual organization's preferences of each user.

\subsection{Integration between Systems}

Our system uses the Facebook API to access specific data using the user's credentials. In our case we need to access data related to the groups created by a specific user. Therefore, during authentication, Facebook asks for consent to use the user_managed_groups permission of the API. Once this permission is granted by the user, it is stored and is never asked again to the user.

The 5 modules are integrated into Moodle in the form of blocks. Each one has to be stored (manually) in a specific Moodle folder in the file system. However, after restart of the system, they are all detected by Moodle, and automatically integrated in registered blocks list. We believe this organization makes the code easier to maintain, and to deploy into another instance of Moodle very easily.

We must stress that the systematic (though expected) evolution of the Facebook API makes the integration with other systems dependant on which permissions are available. It is usual to have a certain permission granted for one version of the API, and in another the right to access that permission is revoked.

\subsection{Authentication}

Once authentication is completed in Moodle, the user needs to authenticate himself in Facebook, using the Facebook authentication mechanism.

This is a persistent mechanism as while the user is logged into Moodle, it does not need to authenticate again in Facebook. The "cookies" created by the system ensure a validation time of 30 days.

Although there is just one pair of credentials to use the API, and to communicate with Facebook, the communicating token is shared by all users of the Moodle system. However, access is only possible to the groups created by that specific user. For example, groups that weren't created, but just subscribed will not be considered by the system. Hopefully this won't 
prevent the further developments we intend to introduce in the system.

\subsection{Using Moodle Modules as Blocks}

Moodle blocks are plugins that load in the right-hand side or left-hand side column of a Moodle site, and display information to the user in a rectangular block. Each side column can have zero, one or multiple blocks loaded. Examples of built-in Moodle blocks include: HTML content, calendars, menus, course lists, etc. A diagram illustrating the standard areas of Moodle is depicted in Figure 2.

Blocks are programmed by extending the PHP class block_base, introducing a init() procedure, and assigning content to the block by using function get_content(). An important advantage of using blocks is that there is already support to "capabilities". Therefore, blocks may invoke the get_context_instance to understand what kind of permissions that particular user has. This allows us to use different accesses to the system (administrator, teacher, students) in very straight forward way. For example, we want to hide the system blocks for nonauthorized access.

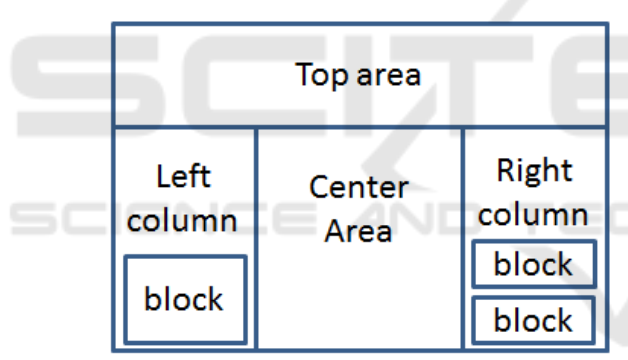

Figure 2: The standard Moodle layout.

Finally, because blocks can be moved in the Moodle standard layout, they can be repositioned during execution time, which makes it easy to the user to choose the most comfortable way of displaying them.

\subsection{Database Tables}

Moodle supports a variety of SQL databases by providing an abstraction layer, based on specific code libraries, which allow the programmer to access every type allowed database in the same way.

Every data table in Moodle has one common integer element: the id. This id is unique in the whole Moodle database which means that it serves as a primary key, but also as a foreign key to any table. This situation is a core rule to Moodle database programming.
Almost all plugins have a hook to the database in the form of a subfolder named ' $d b$ ' that contains files which manage data tables specific to the plugin (or block). The $\mathrm{db}$ folder tipically contains the files install.xml, upgrade.php and access.php. The install.xml defines the tables required for the system (in Listing 1 we present part of install.xml of block fbcomments).

Listing 1: Part of file install.xml.

<?Xml version="1.0" encoding="UTF-8" ?>

<XMLDB PATH="blocks/fbcomments/db" VERSION="20141207" COMMENT="XMLDB file for Moodle blocks/fbcomments/db" xmlns:xsi="http://www.w3.org/2001/XMLSchema-instance" xsi: noNamespaceSchemaLocation="../././lib/xmldb/xmldb .xsd" $>$

$\langle$ TABLES>

<TABLE NAME="fb_user_ids" COMMENT="user identity providers">

$\langle$ FIELDS>

<FIELD NAME="id" TYPE="int" LENGTH="10"

NOTNULL="true" SEQUENCE ="true" />

<FIELD NAME="fbid" TYPE=" char" LENGTH="125"

NOTNULL="true" SEQUENCE $="$ false" $/>$

<FIELD NAME=" courseid" TYPE="char" LENGTH="125"

NOTNULL="true" SEQUENCE $="$ false" $/>$

<FIELD NAME="groupid" TYPE="char" LENGTH="125"

NOTNULL $=$ "true" SEQUENCE $=$ "false" $/>$

<FIELD NAME="groupname" TYPE="char" LENGTH="125"

NOTNULL="true" SEQUENCE="false" />

$\langle/$ FIELDS $\rangle$

〈KEYS>

<KEY NAME="primary" TYPE="primary" FIELDS="id"/> $\langle/$ KEYS $\rangle$

$\langle/ T A B L E\rangle$

The file upgrade.php contains instruction to update table elements, while file access.php defines the capabilities associated with the block.

\begin{tabular}{|l|l|}
\hline \multicolumn{2}{|c|}{ fb_group_stats } \\
\hline K: & group_id:VARCHAR \\
\hline & user_id_mp:VARCHAR \\
\hline & user_id_mc:VARCHAR \\
\hline & user_id_mp_name:VARCHAR \\
\hline & user_id_mc_name:VARCHAR \\
\hline & c_likes:INTEGER \\
\hline & p_likes:INTEGER \\
\hline & n_comments:INTEGER \\
\hline & n_posts:INTEGER \\
\hline & last_event:VARCHAR \\
\hline
\end{tabular}

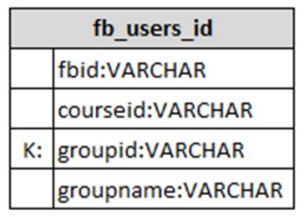

\begin{tabular}{|l|l|}
\hline \multicolumn{2}{|c|}{ fb_appinfo } \\
\hline K: & id:INTEGER \\
\hline & num:INTEGER \\
\hline & appid:VARCHAR \\
\hline & appsecret:VARCHAR \\
\hline
\end{tabular}

\begin{tabular}{|c|l|}
\hline \multicolumn{2}{|c|}{ auth_googleauth2_user_idps } \\
\hline K: & userid:INTEGER \\
\hline & provideruserid:VARCHAR \\
\hline & provider:VARCHAR \\
\hline & accesstoken:VARCHAR \\
\hline
\end{tabular}

Figure 3: EduBridge Social database tables.

The system runs with the support of 4 new database tables, as illustrated in Figure 3. Tables 
fb_group_stats and fb_users_id are used mainly for computing statistics; $\mathrm{fb}_{\text {_appinfo is }}$ used to share Facebook access among users, and; auth_googleauth2_user_idps is used for users' authentication.

Note that although figures, videos and audio do appear in the Moodle block, they are not stored locally. Instead, all these "heavy" media elements are loaded on demand.

\section{INTERFACE}

The system's interface is composed of a four-set Moodle blocks which users, particularly teachers, can display and manage in their Moodle Dashboard (known as "My home" up to Moodle's 2.8 version). This aims at framing the system in the most recent Moodle's philosophy for managing the teacher's educational workspace.

The four blocks are designated as: "Facebook Groups", "Facebook Posts \& Comments", "Facebooks Statistics" and "Total Statistics".

The block's behaviour is interdependent, thought the information displayed on all four blocks is adjusted to users' interaction with the block "Facebook Groups". The "Facebook Groups" block (Figure 4) retrieves the list of groups that the authenticated user manages on Facebook. This means that all educational and personal groups are displayed and accessible through Moodle's interface. This is also the main block among the four-set, since it allows teachers to arrange the information and views to be generated.

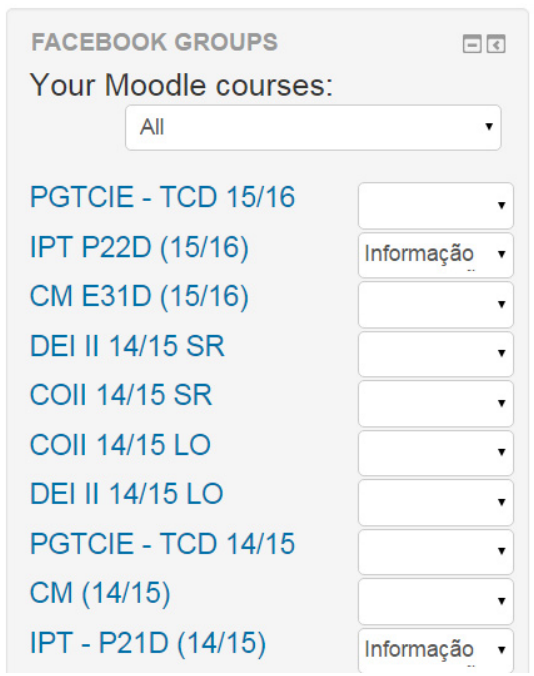

Figure 4: "Facebook Groups" block.
In order to provide teachers with tools to (1) separate personal from educational groups and (2) arrange and match the groups to their courses on Moodle a set of dropdown matching boxes were incorporated.

The ability to match Facebook Groups to Moodle courses is also aimed at providing teachers with greater flexibility to accommodate the need for diversity of educational scenarios, concerning the arrangement of students in wider or narrower social learning networks. Assigned to the same Moodle course it is possible to have one or more Facebook groups, whether the teacher intends to arrange the class into separate visible or non-visible teams. This is also aimed at providing teachers with the same Moodle course participants' management features, concerning the creation of course level groups.

As illustrated on Figure 4, a dropdown box presents teachers the full list of his/her own Moodle courses, which can be assigned to each Facebook's group. Having students arranged into separate Facebook groups will allow teachers to monitor each group's performance individually and to assess each group's participant commitment and contributions to the assigned projects/activities, which is displayed on the "Facebook Statistics" and "Total Statistics" block. Figure 4 presents a real case scenario in which the teacher only uses one Facebook group per Moodle course. However, it is possible to have other grouping configurations, such as the need to create several Facebook groups belonging to the same Moodle course, thus this feature was added the "Facebook Groups" module. This allows the analysis of interactions developed in separate groups that belong to the same class/course, particularly in courses in which students are assigned concurrent and separate projects development and in individual / group assessment is needed.

The system also provides teacher with the ability to access all the group's posts and interactions on Moodle's Dashboard, through the block "Facebook Posts and Comments" (Figure 5). This block's main purpose is to increase the level of integration between the two platforms, since it eliminates the need to access a second environment (Facebook) in order to check updates and new user's interactions, which may or may not require action.

It is recommended that this block is displayed on the Dashboard's central area, as illustrated in Figure 2 , given the amount and of information it provides, which includes every posts' text and other visual static or dynamic multimedia content. 


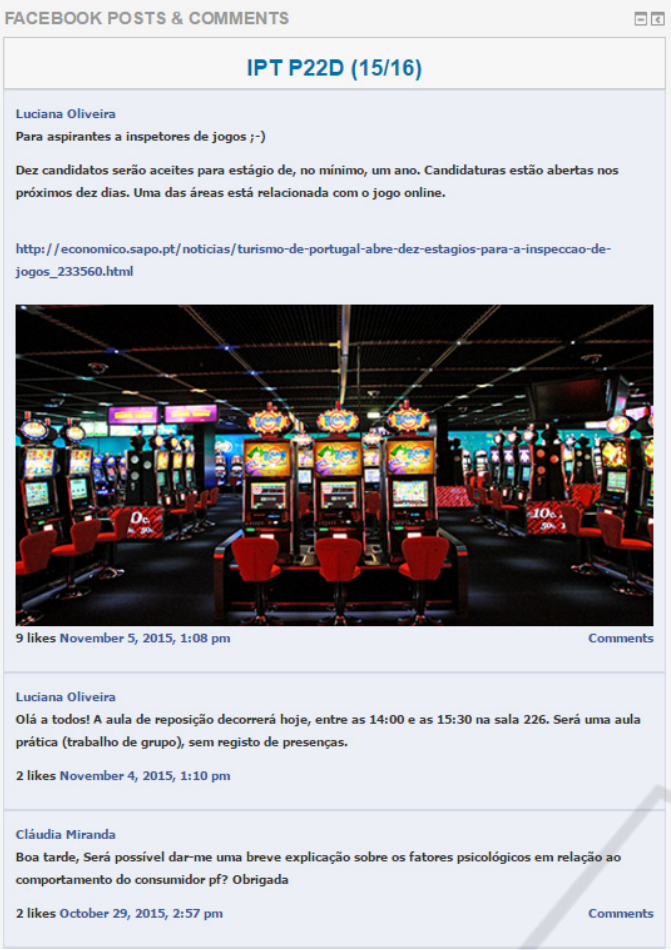

Figure 5: "Facebook Posts and Comments" block.

For each post it is possible to access the post author, post date, time and post comments. For the sake of economy of page height, comments are collapsed by default, and can be further expanded by the user, as illustrated in Figure 6.

For each comment, the block displays the comment author, date, time, likes, comment's likes and replies.

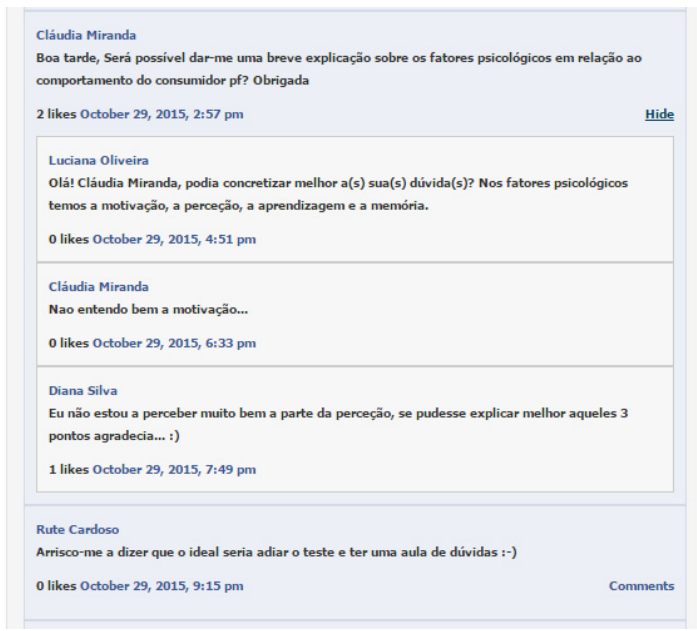

Figure 6: "Facebook Posts and Comments" block with expanded comments view.
The "Group Statistics" block presents teachers statistical data concerning the group's total number of posts, total number of posts, median of comments per post, retrieves the group's most recent post and comment and also the most popular post, which is the post with more likes, as illustrated in Figure 7. This block also displays user interaction statistics, providing relevant information for student's performance assessment, as illustrated in Figure 8.

Statistical information about student's performance is organized in two sections: "Users who posted" new messages in the group and the corresponding amount of likes and "Users who commented", that is, the users who interacted with the messages posted on the same group, by posting replies of liking comments.

Users (teacher and students) are ranked by the amount of interaction they generate in the group. Rankings of users' statistical data are particularly useful during the teaching-leaning process and specifically relevant for student continuous or final assessment.

For instance, a user may post a lot of messages in the group, but may not receive feedback at all (that is, the content may not be relevant for that community) and / or may not interact at all with other users

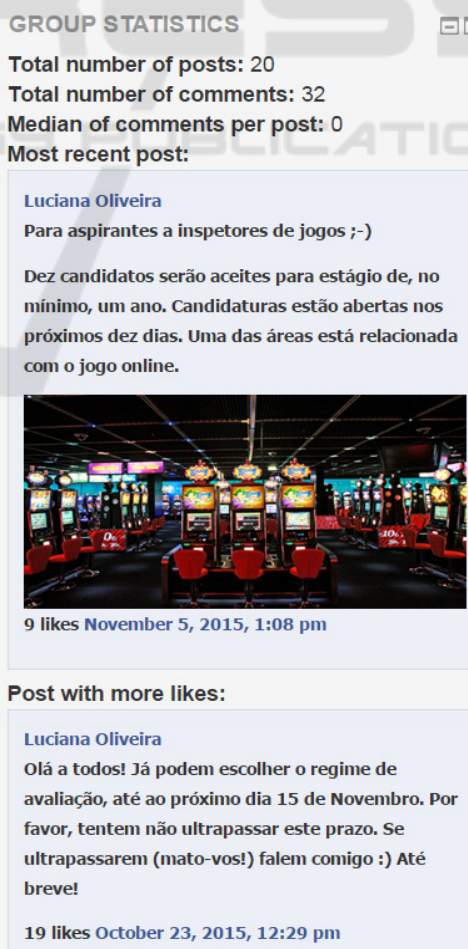

9 likes November 5, 2015, 1:08 pm

Post with more likes:

Luciana Oliveira

Olá a todos! Já podem escollher o regime de avaliação, até ao próximo dia 15 de Novembro. Por favor, tentem não ultrapassar este prazo. Se ultrapassarem (mato-vos!) falem comigo :) Até breve!

19 likes October 23, 2015, 12:29 pm

Figure 7: "Group Statistics" block (a). 
messages (that is, he / she may not find other user's content relevant and / or this may be an indicator of lack of interest, inexistence of group sense of belonging, etc.).

On the other hand, a user may not introduce any content at all in the group, but he / she may be an active and valuable member to the community, either by commenting, replying to comments (i.e., fostering discussion) or liking his / her colleagues' messages.

It is up to the teacher to consider the weight of these parameters in assessing individual or group performance (is assessment is applicable).

The fourth block, "Facebook Total Statistics" (Figure 9), displays the global statistics for the Moodle course selected on the "Facebook Groups" block. In case two or more Facebook groups are assigned to the same Moodle course, this block will

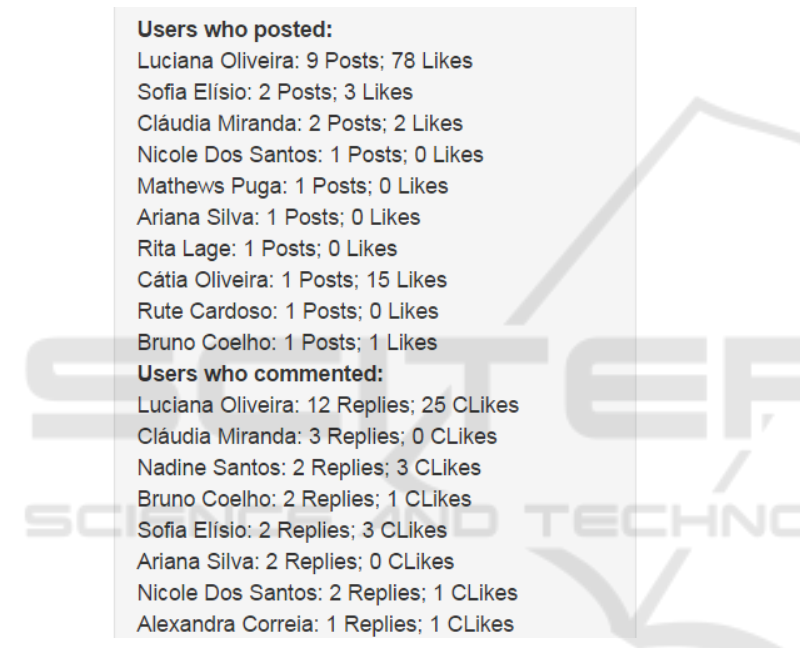

Figure 8: "Group Statistics" user's interactions section (b).

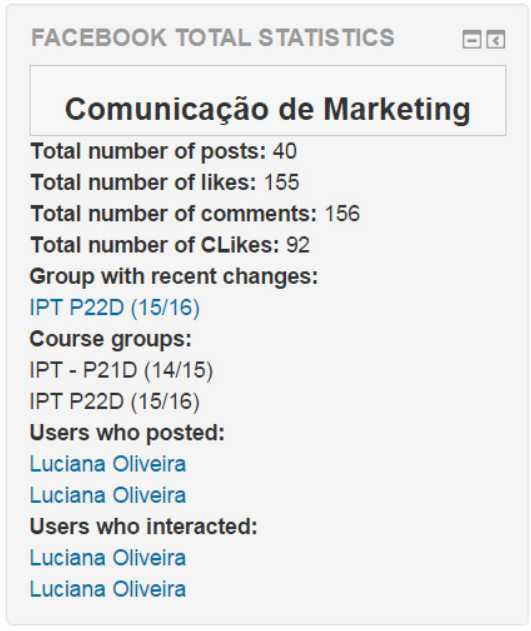

Figure 9: "Facebook Total Statistics" block. provide the teacher the aggregated statistics for that set of groups. This feature is particularly relevant when the teacher intends to have the class divided into groups and uses several Facebook groups to guide and support each group separately.

This block is also useful for teacher needing aggregated statistics for all his / her Facebook groups of for personalized sets of groups, which are matched in the "Facebook Groups" block.

\section{DISCUSSION}

The proposed methodology, previous research outputs and presented system allowed for the development and implementation of "How to use Facebook groups in education? advantages and limitations" workshops. The workshops were conducted by the researchers and were aimed at teachers' pedagogical and technological development and diversification. A lack of pedagogical validity was detected during this dissemination phase, which lead us to the need of incorporating new features in the already established LMS (Moodle). A very relevant detail, which is not frequently mentioned in literature, and is of the utmost importance to this scenario, is the discrepancy between the pace of research and its concrete / tangible corresponding transfer into the educational context. In some organizational cultures, the proneness to adopt or adapt to new pedagogical methodologies / technologies is very slow. This was recognized by researchers during the initial stages of introduction of institutional LMS in education (Rogers 2010), when teachers' proneness to adopt technology mediated education was a hot topic. This means that, despite the advances in research, priorities should also rely on the academic and day-to-day practices, thus it is not our intent to contribute to the persistent gap between researchers and practioners. Our main goal, is to capacitate and actually facilitate the adjustment of teachers to new tendencies. In our perspective, this can only be accomplished by capacitating the wellknown environments with new features, reducing the notion of risk that teachers often associate to pedagogical innovation.

\section{CONCLUSIONS AND FUTURE WORK}

In this paper we described and discussed the concept of "Social Student Relationship Management" as an emergent need in the context of social media applied 
to educational environments. We describe the motivation beyond this need and its benefits. We, then, focus on the Facebook social network and on its groups as a privileged environment for discussions, much in the same line as forums, however, with much more flexibility. We stressed the importance of "social media" groups, relating a case study using Facebook and reporting the results.

These results led us to create a system which bridges Moodle with Facebook. The proposed architecture is based on the model-view-controller and can be applied to most social networks with minimal effort. We described the system's user interface with several examples taken from its real usage.

Future technical development includes both the optimization of the Moodle blocks presented in this paper, and the addition of relevant learning analytics for teachers and for students, built upon social media data and metrics. Learning analytics is a current hot topic which we aim to address on the teacher and student perspective, aiming at providing a validated tool for formal and informal learning assessment.
Dorin, B., Razvan, B., \& Bogdan, P. (2013). The Role of the Web Technologies in Connection to the Communication's Streamlining and Diversification between the Actors of a Learning System. In P. Monica \& P. Bogdan (Eds.), Social Media in Higher Education: Teaching in Web 2.0 (pp. 216-236). Hershey, PA, USA: IGI Global.

Oliveira, L. (2015). Social Student Relationship Management in Higher Education: extending educational and organisational communication into Social Media. 9th Annual International Technology, Education and Development Conference, IATED.

Palloff, R. M., \& Pratt, K. (2007). Online learning communities in perspective. Online learning communities, 3-15.

Parsons, T., \& Halsey, A. (2014). The school class as a social system. Schools and Society, 35.

Reuben, R. (2008). The use of social media in higher education for marketing and communications: A guide for professionals in higher education. EduGuru.

Rogers, E. M. (2010). Diffusion of innovations, Simon and Schuster.

Wang, Q., Woo, H. L., Quek, C. L., Yang, Y., \& Liu, M. (2012). Using the Facebook group as a learning management system: An exploratory study. British Journal of Educational Technology, 43(3), 428-438.

\section{ACKNOWLEDGEMENTS}

This work is supported by the ERDF - European Regional Development Fund through the COMPETE Programme (operational programme for competitiveness) and by National Funds through the FCT (Portuguese Foundation for Science and Technology) within project «Reminds/ UTAPICDT/EEI-CTP/0022/2014.

\section{REFERENCES}

Bruckman, A. (2006). Learning in online communities. K.R. Sawyer (Ed.), The Cambridge handbook of the learning sciences, Cambridge University Press, New York (2006), pp. 461-472.

Bryant, V.C., et al., School as heaven: Transforming school environments into welcoming learning communities. Children and Youth Services Review, 2013. 35(5): p. 848-855.

Cheung, W. S., Hew, K. F., \& Ng, C. S. L. (2008). Toward an understanding of why students contribute in asynchronous online discussions. Journal of Educational Computing Research, 38(1), 29-50.

Deng, L., \& Tavares, N. J. (2013). From Moodle to Facebook: Exploring students' motivation and experiences in online communities. Computers \& Education, 68, 167-176. doi: http://dx.doi.org/ 10.1016/j.compedu.2013.04.028. 